\title{
Therapy for thoracic lumbar and sacral vertebrae tumors using total spondylectomy and spine reconstruction through posterior or combined anterior-posterior approaches
}

\author{
PINGLIN YANG, XIJING HE, HAOPENG LI, QUANJIN ZANG and GUOYU WANG \\ Second Department of Orthopedics, Second Affiliated Hospital of Xi'an Jiaotong University Medical School, \\ Xi'an, Shaanxi 710004, P.R. China
}

Received July 24, 2014; Accepted April 8, 2015

DOI: $10.3892 / 01.2016 .4126$

\begin{abstract}
The present study aimed to analyze the indications, feasibility, safety and clinical effects of total spondylectomy and spine reconstruction through posterior or combined anterior-posterior approaches for thoracic lumbar and sacral vertebrae tumors. Between December 2009 and May 2012, 10 patients with thoracic lumbar and sacral vertebrae tumors were retrospectively analyzed. Different surgical indications and approaches were used according to the affected segments, the extent of lesion involvement and the specific pathology results. One-stage posterior or combined anterior-posterior total spondylectomy and reconstruction was used for the treatment of complicated thoracic lumbar and sacral vertebral malignant tumors and invasive benign tumors. The duration of surgery, levels of intraoperative blood loss and transfusions, and the clinical effects were observed. The average surgical duration was $6.8 \mathrm{~h}$ (range, $4.8-12 \mathrm{~h}$ ), with an average blood loss level of 3,200 $\mathrm{ml}$ (range, 1,500-10,000 ml) and an average transfusion level of 2,500 $\mathrm{ml}$. During the average 15 months (range, 3-29 months) follow up, two patients succumbed and one patient experienced tumor recurrence. Neither tumor reoccurrence nor metastasis was observed in all other patients. Personalized surgical indications and approaches according to the affected segments, the extent of lesion involvement and the specific pathology results would aid in the reduction of pain, the improvement of nerve function and the reduction of tumor recurrence.
\end{abstract}

Correspondence to: $\mathrm{Dr}$ Xijing $\mathrm{He}$, Second Department of Orthopedics, Second Affiliated Hospital of Xi'an Jiaotong University Medical School, 157 West Fifth Road, Xi'an, Shaanxi 710004, P.R. China

E-mail: xijing_h@vip.tom.com

Key words: spinal tumor, total spondylectomy, posterior, combined anterior-posterior

\section{Introduction}

Tumors of the spine are defined as an abnormal mass of tissue within or surrounding the spinal cord or spinal column (1). Spine tumors may be characterized according to the location in which they occur, such as the lumbar and sacral regions. The majority of spinal tumors have an unknown cause, however, exposure to cancer-causing agents may be involved (2). The most frequent symptom of spinal tumors is non-mechanical back pain, particularly in the middle or lower back (2). Usually a combination of radiological and medical examinations, which focus on back pain and neurological deficits, are used to diagnose tumors of the spine (3). At present, both surgical and nonsurgical treatments are used for the treatment of spinal tumors, however, treatment outcome is dependent on various factors, such as patient age, overall health of the patient and whether the tumor is benign or malignant and primary or metastatic (3). The majority of spinal tumors are metastatic, and are usually treated with palliative therapy, including radiotherapy, chemotherapy and selective artery embolism. However, chemotherapy and radiotherapy have extremely limited effects in the treatment of spine tumors (4). Selective embolization may be used to control tumor size and reduce bleeding during surgery (5). Approximately $5 \%$ of spine tumors are primary tumors (6) and surgical treatment can be used to completely cure such tumors in certain cases. It has also been reported that surgery may be used to relieve pain in $80-90 \%$ of patients with malignant spinal tumors, as well as improving spinal function in $75 \%$ of patients (4).

Spondylectomy for the treatment of spinal neoplasms was first reported by Stener in 1971 (7) and further used by Roy-Camille et al (8) and Fidler (9). In the 1990s, Tomita et al (10) developed and popularized the surgical procedure known as total en bloc spondylectomy (TES) to resect the diseased vertebrae. The TES procedure has been increasingly gaining recognition and is now widely accepted by spinal tumor surgeons due to its favorable outcomes $(11,12)$.

Generally there are two surgical steps to the TES procedure $(10,13,14)$ : Step one is a total-laminectomy, which involves the excision of the posterior of the vertebrae, and step two is the resection of the total front of the vertebral 
Table I. Data on patients with primary malignant tumors of the spine.

\begin{tabular}{|c|c|c|c|c|c|c|c|}
\hline $\begin{array}{l}\text { Patient } \\
\text { no. }\end{array}$ & Diagnosis & Age, years & Levels & $\begin{array}{c}\text { Surgical } \\
\text { duration, h }\end{array}$ & $\begin{array}{l}\text { Intraoperative } \\
\text { blood loss, } \mathrm{ml}\end{array}$ & Status & $\begin{array}{c}\text { Follow-up, } \\
\text { months }\end{array}$ \\
\hline 1 & Giant cell tumor & 23 & L5-S2 & 11 & 4000 & NED & 24 \\
\hline 2 & Giant cell tumor & 44 & L3-4 & 7 & 3200 & NED & 22 \\
\hline 3 & $\begin{array}{l}\text { Malignant fibrous } \\
\text { histiocytoma }\end{array}$ & 36 & T6 & 5 & 2300 & DOD & 8 \\
\hline 4 & Chordoma & 66 & S3 or below & 3 & 1500 & NED & 28 \\
\hline 5 & Chordoma & 59 & S3 or below & 4 & 1800 & DOC & 18 \\
\hline 6 & Chordoma & 65 & S3 or below & 3 & 1600 & NED & 30 \\
\hline 7 & Plasma cell myeloma & 59 & $\mathrm{~T} 8$ & 5 & 2800 & NED & 36 \\
\hline 8 & Plasma cell myeloma & 62 & $\mathrm{~T} 7$ & 6 & 2400 & NED & 18 \\
\hline 9 & Giant cell tumors & 22 & L3 & 8 & 10000 & NED & 33 \\
\hline 10 & Chondrosarcoma & 46 & $\mathrm{~T} 7$ & 5 & 1800 & NED & 36 \\
\hline
\end{tabular}

NED, no evidence of disease; AWD, alive with disease; DOD, died of disease; DOC, died of other causes.

body. The present study reports 10 cases of total spondylectomy and spine reconstruction through posterior or combined anterior-posterior approaches for thoracic lumbar and sacral vertebral tumors.

\section{Patients and methods}

Patient data. Between December 2009 and May 2012, 10 cases of patients with primary malignant tumors of the spine treated by TES in the Second Affiliated Hospital of Xi'an Jiaotong University Medical School (Xi'an, China) were retrospectively analyzed (Table I). The average age of the patients at the time of surgery was 50.5 years (range, 23-65 years), with an average follow-up time of 50.2 months (range, 28.1-68.7 months). The study group consisted of three females and seven males.

All 10 cases were of primary tumors, with three chordomas, three giant cell tumors of the bone, two plasma cell myelomas, one chondrosarcoma and one malignant fibrous histiocytoma. Affected segments were T6, T8 and L3 in one case each, T7 in two cases, L3-4 in one case, L5 and S1-2 in one case each, and S3 or below in three cases. According to Tomita's grading system (15), there were four type IV cases, four type V cases and two type VI cases. Using the Frankel classification of pre-operative spinal cord function (16), one case was grade A, two cases were grade $\mathrm{C}$, two cases were grade $\mathrm{D}$ and five cases were grade E. Seven cases required a partial spondylectomy and three required a total spondylectomy. According to the recorded data for the height, weight and body mass index of the patients, the average body mass index was $25.7 \mathrm{~kg} /$ $\mathrm{m}^{2}$ (range, $22.2-39.5 \mathrm{~kg} / \mathrm{m}^{2}$ ). Written informed consent was obtained from all patients and this study was approved by the ethics committee of the Second Affiliated Hospital of Xi'an Jiaotong University Medical School.

TES procedure. The TES procedure generally consists of two steps: Step one is a total-laminectomy, which involves the excision of the posterior of the vertebrae, and step two is the resection of the total front of the vertebral body. In step one, the surgical area is focused on the lesion centrum and the extension for one centrum. When the transverse of the lesion centrum is fully exposed, the bilateral pedicle is cut off. The T-saw guide is carefully inserted into the middle of the lamina and vertebral pedicle surface, and removed from the neural tube, to completely resect the bilateral pedicle and avoid nerve root damage. The guide is removed following the introduction of a special wire saw, which is used to cut off the vertebral pedicle. To reduce bleeding and contamination by tumor cells, the cut off vertebral pedicle is wrapped with gauze. The posterior spine is fixed by temporary equipment to maintain spinal stability following resection. The bilateral segmental artery is carefully identified and protected prior to step two. The thoracic vertebra nerve root is cut off in the intervertebral foramen. The segmental artery branch along the nerve root is also cut off. Next, a blunt dissection is performed between the pleura (or iliopsoas) and the centrum. The aorta is separated from the anterior centrum using a curette and fingers. The intervertebral disc is cut carefully using the wire saw. The dura mater is then separated from the venous plexus and ligaments in the surrounding spinal canal by nerve dissection. Finally, the anterior and posterior of the centrum are removed.

Surgical approach. According to the affected segments, the extent of lesion involvement and the specific pathology results, different surgical indications and approaches were selected. A one-stage posterior or combined anterior-posterior total spondylectomy and reconstruction was used for the treatment of complicated thoracic lumbar and sacral vertebral malignant and invasive benign tumors. Titanium mesh autologous bone grafts were used for intervertebral fusion. The indications for a total spondylectomy were nerve function defects, an inability to withstand back pain medication due to spinal instability, or pain that could not be reduced by injections.

For the affected segments located at the thoracic level and L3 or above (not including L3 spine), as the tumor 

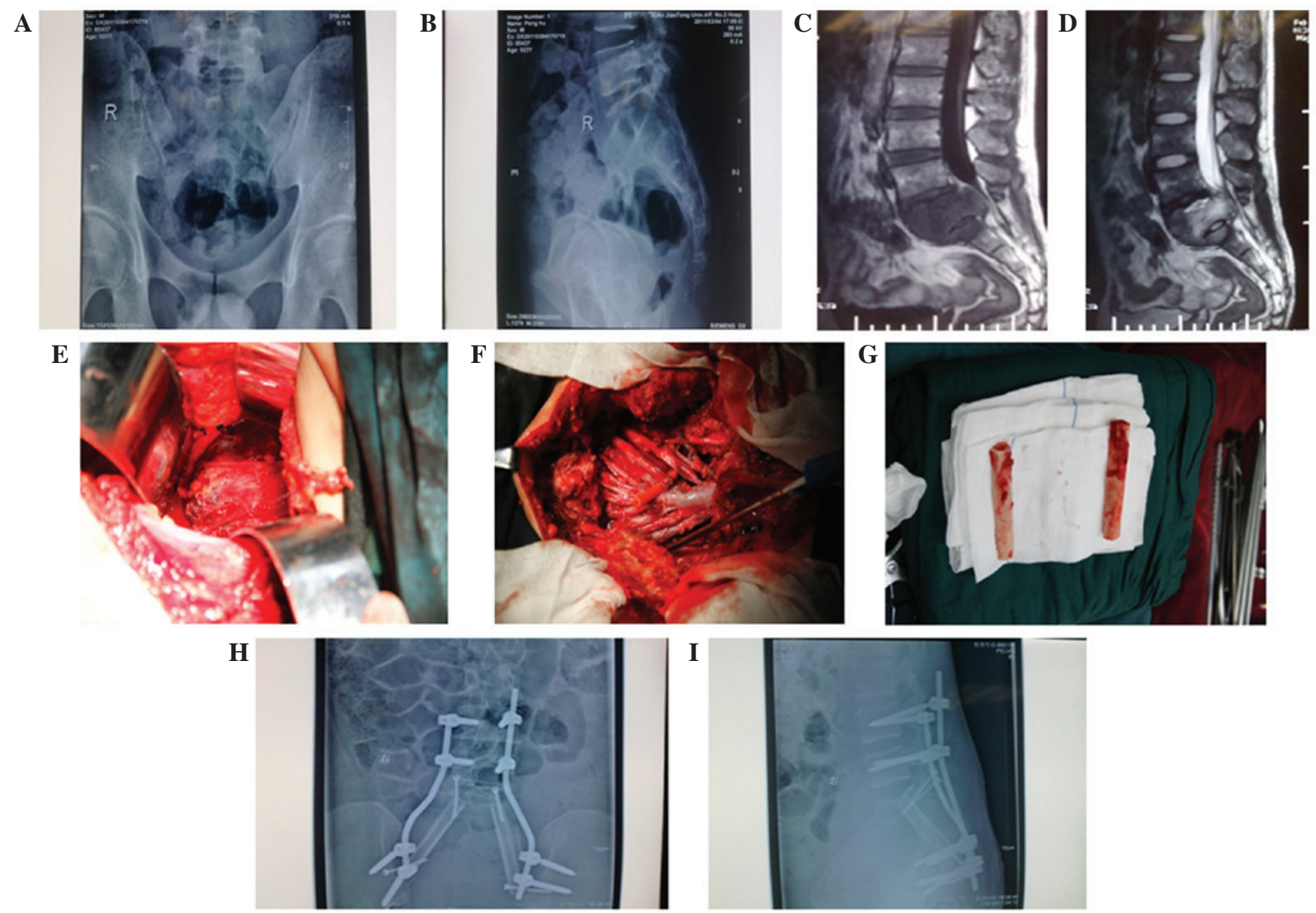

Figure 1. Giant cell tumor at L5-S2 in a 23-year-old male. (A) Positive and (B) lateral X-ray films of the sacrum, and (C) T1 and (D) T2 sagittal-weighted magnetic resonance pre-operative imaging showing the tumor expanding into the spinal canal. (E and F) Intraoperative view of a tumor in L5-S2 and (G) bilateral posterior reconstruction of an autologous fibula. Post-operative radiograph $(\mathrm{H})$ positive and (I) lateral views of the lumbar spine at 24 months post-surgery showing that the reconstructed lumbar spine was well maintained.

did not invade into the main blood vessels, a one-stage posterior partial spondylectomy and titanium cage bone graft was used, together with a posterior screw rod system reconstruction. For S3 or below, a one-stage posterior partial spondylectomy without reconstruction was used. For L3-5, a one-stage anterior/posterior partial spondylectomy, spinal canal decompression, a posterior screw rod system and a titanium cage bone graft reconstruction were applied. Lastly, for L5-S2, a one-stage anterior/posterior partial spondylectomy, spinal canal decompression, a posterior screw rod system and a bilateral autologous fibula reconstruction were applied.

Data collection. All patients were followed-up on months 1, 3,6 and 12 post-surgery, and then once a year thereafter. The follow-up evaluation was performed using medical imageology analysis by spine specialists who had not been involved in treating the patients. The frontal and lateral $\mathrm{X}$-rays and computed tomography (CT) were reviewed prior to or following the surgery. X-ray analysis, including the measurement of body weight and segmental angle, the collapsed or displaced vertebral plate grid, and any spinal column instability or misalignment. CT scan was used to detect the vertebral plate grid of the graft bone, and at least two or more sagittal section images were included with adjacent vertebral graft bone bridge plates.

\section{Results}

Surgical and patient data. The average surgical duration was $6.8 \mathrm{~h}$ (range, $4.8-12 \mathrm{~h}$ ) with an average level of blood loss of 3,200 ml (range, 1,500-10,000 ml). All patients were followed-up for an average of 15 months (range, 3-29 mouths). Two patients succumbed and one patient experienced tumor recurrence at follow-up. One-stage posterior total spondylectomy for lesions in the thoracic vertebrae or L3 and above showed thorough removal of the lesional focus and immediate stability of the spine without fixation, break or evident complication.

At six months post-operatively, the spinal cord function for all patients recovered to Frankel classification grade E. One patient with a lesion in $\mathrm{S} 3$ or below showed recurrence, with urination and defecation function disturbance. One patient with a L3-5 lesion treated with combined posteroanterior total spondylectomy showed decreased bilateral quadriceps muscle strength from level 4 to level 2, complicated by radiating pain in the hip. However, X-rays and magnetic resonance examination showed thorough decompression, good spinal stability and no internal fixation loosening or fracture during the follow-up. One patient with a L5-S2 lesion showed partial paralysis of the lower limbs, and spinal cord function decreased from grade D to B. Following one year of 
follow-up, bone graft healing without tumor recurrence was observed, but the posterior screw-rod was broken.

Sample case. One of the patients treated was a 23-year-old male with a giant cell tumor (case 1 in Table I). The tumor expanded into the spinal canal and outside of the vertebral body (Fig. 1). Bilateral posterior reconstruction with an autologous fibula was used. Post-operative radiographs and CT scans of the lumbar spine suggested that the reconstructed spine was well maintained (Fig. 1).

\section{Discussion}

Total spondylectomy is one of the most difficult surgeries to perform for the treatment of upper thoracic vertebral tumors with the advantage of the complete removal of tumors (17). One-stage posterior total spondylectomy and spine reconstruction can be used to treat primary malignant tumors in the spine, including chordoma, osteosarcoma and chondrosarcoma. In addition, benign upper thoracic vertebra tumors with the potential for invasion or a higher recurrence rate, such as giant cell tumors, can also be treated by TES (18). Other tumors that lead to nerve dysfunction and compression fractures, as well as those metastatic tumors in a single vertebra also require TES. The Second Affiliated Hospital of Xi'an Jiaotong University Medical School has successfully treated a number of cases of total spondylectomy with satisfactory results.

Different techniques for en bloc resection have previously been described (8-10,19-21). To achieve a total spondylectomy and reconstruction, a combined anterior-posterior surgical approach has usually been used. However, this approach can lead to more serious injury, a longer surgical duration and greater blood loss, as well as increasing the psychological and economic burden on the patient. The frontal thoracotomy approach requires greater patient tolerance and may lead to the injury of the visceral pleura or large blood vessels during surgery. The approach has increased risks of atelectasis and pulmonary infection, as well as an increased risk of respiratory failure post-operatively for elderly patients with poor lung function and lung diseases, or in those with pleural adhesion or who underwent open heart surgery (22).

By contrast, surgery using the posterior approach could avoid intraoperative injury and the corresponding risk of complications (23). This approach does not require a thoracotomy, and is therefore suitable for more patients as it requires a lower level of tolerance, reduces the surgical trauma, shortens the surgical duration, lowers the level of intraoperative blood loss, relieves the patients' pain, reduces medical costs and shorten the hospital stay. However, the posterior approach also requires doctors with higher surgical skills, due to the narrow filed of vision, limited surgical space and easily damaged spinal cord.

Therefore, we believe that the indications for a posterior thoracic vertebra total spondylectomy and reconstruction are as follows: Primary malignant spinal tumors and aggressive benign tumors; tumors without front internal organ invasion; tumors without adhesion to the inferior vena cava and aorta; no multiple metastases; less than 3 vertebral body lesions; and an isolated vertebral metastatic tumor, without primary lesion, or a primary tumor lesion under control. The present results showed that survival time can be extended to between three months and half a year with the surgery, and the quality of life can be significantly improved.

Overall, the following conclusions can be made from this study: Firstly, if the lesion location is above L3 without main venous invasion, the posterior approach only gives a better result. Secondly, to treat tumors with main venous invasion or serious segmental blood vessel adhesion, the anterior approach should be used first, followed by a posterior total spondylectomy and reconstruction. Lastly, for those lesions located at L3-S2, where the surgical field may be covered by the ala ossis ilii, and affected by the existence of abdominal aortic bifurcation and psoas major, the use of a combined anterior and posterior approach would result in a better prognosis.

\section{References}

1. Dahlin DC (ed): Bone Tumors; General Aspects and Data on 3,987 cases. 2nd edition. C. C. Thomas, Springfield, IL, p285, 1967.

2. Van Goethem JW, van den Hauwe L, Ozsarlak O, De Schepper AM and Parizel PM: Spinal tumors. Eur J Radiol 50: 159-176, 2004.

3. Bilsky MH, Lis E, Raizer J, Lee H and Boland P: The diagnosis and treatment of metastatic spinal tumor. Oncologist 4: 459-469, 1999.

4. Frymoyer JW and Wiesel SW (eds). The Adult and Pediatric Spine. 3rd edition. Lippincott Williams \& Wilkins, Philadelphia, PA, 2004

5. Simmons ED and Zheng Y: Vertebral tumors: Surgical versus nonsurgical treatment. Clin Orthop Relat Res 443: 233-247, 2006.

6. Munoz-Bendix C, Slotty PJ, Ahmadi SA, Bostelmann R, Steiger HJ and Cornelius JF: Primary bone tumors of the spine revisited: A 10-year single-center experience of the management and outcome in a neurosurgical department. J Craniovertebr Junction Spine 6: 21-29, 2015.

7. Stener B: Total spondylectomy in chondrosarcoma arising from the seventh thoracic vertebra. J Bone Joint Surg Br 53: 288-295, 1971.

8. Roy-Camille R, Saillant G, Mazel CH and Monpierre H: Total vertebrectomy as treatment of malignant tumors of the spine. La Chir Organi Mov 75 (1 Suppl): 94-96, 1990.

9. Fidler MW: Radical resection of vertebral body tumours. A surgical technique used in ten cases. J Bone Joint Surg Br 76: 765-772, 1994

10. Tomita K, Kawahara N, Baba H, Tsuchiya H, Fujita T and Toribatake Y: Total en bloc spondylectomy. A new surgical technique for primary malignant vertebral tumors. Spine (Phila $\mathrm{Pa}$ 1976) 22: 324-333, 1997.

11. Kato S, Murakami H, Demura S, et al: More than 10-year follow-up after total en bloc spondylectomy for spinal tumors. Ann Surg Oncol 21: 1330-1336, 2014.

12. Tomita K, Kawahara N, Murakami H and Demura S: Total en bloc spondylectomy for spinal tumors: improvement of the technique and its associated basic background. J Orthop Sci 11: $3-12,2006$

13. Kawahara N, Tomita K, Murakami H, Demura S, Yoshioka K and Kato S: Total en bloc spondylectomy of the lower lumbar spine: a surgical techniques of combined posterior-anterior approach. Spine (Phila Pa 1976) 36: 74-82, 2011.

14. Demura S, Kawahara N, Murakami H, et al: Total en bloc spondylectomy for spinal metastases in thyroid carcinoma. J Neurosurg Spine 14: 172-176, 2011

15. Tomita K, Kawahara N, Kobayashi T, et al: Surgical strategy for spinal metastases. Spine (Phila Pa 1976) 26: 298-306, 2001.

16. Ditunno JF Jr, Young W, Donovan WH and Creasey G: The international standards booklet for neurological and functional classification of spinal cord injury. American Spinal Injury Association. Paraplegia 32: 70-80, 1994.

17. Guo C, Yan Z, Zhang J, et al: Modified total en bloc spondylectomy in thoracic vertebra tumour. Eur Spine J 20: 655-660, 2011.

18. Chung JY, Kim SK, Jung ST and Lee KB: New posterior column reconstruction using titanium lamina mesh after total en bloc spondylectomy of spinal tumour. Int Orthop 37: 469-476, 2013. 
19. Boriani S, Biagini R, De Iure F, et al: En bloc resections of bone tumors of the thoracolumbar spine. A preliminary report on 29 patients. Spine (Phila Pa 1976) 21: 1927-1931, 1996.

20. Krepler P, Windhager R, Bretschneider W, Toma CD and Kotz R Total vertebrectomy for primary malignant tumours of the spine. $\mathrm{J}$ Bone Joint Surg Br 84: 712-715, 2002.

21. Mazel C, Grunenwald D, Laudrin P and Marmorat JL: Radical excision in the management of thoracic and cervicothoracic tumors involving the spine: results in a series of 36 cases. Spine (Phila $\mathrm{Pa}$ 1976) 28: 782-792, 2003.
22. Yang Q, Li JM, Yang ZP, Li X, Li ZF and Yan J: Treatment of thoracolumbar tumors with total en bloc spondylectomy and the results of spinal stability reconstruction. Zhonghua Zhong Liu Za Zhi 35: 225-230, 2013 (In Chinese).

23. Matsumoto M, Tsuji T, Iwanami A, et al: Total en bloc spondylectomy for spinal metastasis of differentiated thyroid cancers: a long-term follow-up. J Spinal Disord Tech 26: E137-E142, 2013. 